Background and Aim Although hypoalbuminemia has been recognized as a marker of poor outcome in adult patients, this association has not been demonstrated in a general population of pediatric critically ill children, and studies have not considered non-nutritional factors that may influence albumin concentrations. This study aimed to determine whether hypoalbuminemia is associated with mortality and morbidity of critically ill children while considering the clinical severity of the patients.

Methods This was a prospective study involving 178 children admitted to the ICU. The outcome variables studied were ICU mortality, severity of organ dysfunction, free-ventilator days and freeICU days. The outcome variables were as follows: nutritional status, Pediatric Index of Mortality (PIM 2), serum albumin, C Reactive Protein and lactate concentrations. Children with liver failure and chronic kidney disease were excluded.

Results Mean serum albumin concentration upon admission among survivors was $3.16+/-0.66$ versus $2.63+/-0.67$ in non-survivors $(\mathrm{p}=0.015)$. The mortality rate was $6.4 \%(11 / 178)$. In a multiple logistic regression model, adjusting for PIM 2 score, lower albumin concentrations were independently associated with increased organ dysfunction (OR: 0.18, 95\% CI: 0.06-0.53; p=0.002) and mortality (OR: $0.22,95 \%$ CI: $0.07-0.76 ; \mathrm{p}=0.017$ ). In a multiple linear regression model, adjusted for PIM2, malnutrition and other potential confounders, lower albumin concentrations were associated with fewer free-ventilator days $(\mathrm{p}=0.024)$ and free-ICU days $(\mathrm{p}=0.028)$.

Conclusions Children with hypoalbuminemia at admission are at a greater risk of organ dysfunction and mortality, and longer time of mechanical ventilation and length of ICU stay, independent of clinical severity and nutritional status.

\section{BEDSIDE C-REACTIVE PROTEIN TESTING IN FEBRILE CHILDREN REDUCES LENGTH OF STAY AT THE EMERGENCY DEPARTMENT}

doi:10.1136/archdischild-2012-302724.0826

'RG Nijman, 'R Oostenbrink, ${ }^{2} Y$ Vergouwe, ${ }^{1} Y$ van lerland, ${ }^{3} Y$ De Rijke, ${ }^{1} H A$ Moll. 'Department of General Paediatrics, Erasmus MC - Sophia Children's Hospital; ${ }^{2}$ Center for Medical Decision Making, Erasmus MC; ${ }^{3}$ Department of Clinical Chemistry, Erasmus MC - Sophia Children's Hospital, Rotterdam, The Netherlands

Background and Aims C-Reactive Protein (CRP) is an important diagnostic marker in the evaluation of febrile children. Aim is to study if bedside CRP testing reduces the length of stay (LOS) of febrile children at the emergency department (ED).

Methods We conducted a before-after study of previously healthy children with fever, aged 1 month to 16 years, who attended the paediatric ED of the Erasmus MC - Sophia, Rotterdam, The Netherlands, between 2008-2011. Bedside CRP testing was implemented in 2009, while conventional CRP testing remained optional. We used multivariable linear regression analysis to study the effect of introducing bedside CRP testing on (log transformed) LOS at the ED.

Results In the pre-implementation cohort we included 651 children; 319 (49\%) had a conventional CRP ordered at the discretion of the physician. In the post-implementation cohort we included 1376 children; 703 (51\%) had bedside CRP and 223 (16\%) conventional CRP testing. Bedside CRP reduced the median LOS to 148 minutes (interquartile range (IOR): 109-201); in the pre-implementation cohort this was 176 minutes (IOR: 132-231) ( $p<0.001)$. LOS of children with conventional CRP was similar between the two cohorts. Other important determinants of (log)LOS were hospitalisation (beta: 0.32 (se: 0.04), p<0.001), transfer to a different hospital $(0.53$ $(0.04), p<0.001)$ and shift of presentation (daytime: $0.25(0.03)$, $\mathrm{p}<0.001$; evening: 0.19 (0.03), $\mathrm{p}<0.001)$; night = reference).

Conclusion CRP bedside testing substantially reduces LOS at the ED for children with fever. Other important determinants of LOS were hospitalisation and time of presentation.

\section{EVOLUTION OF OTITIS MEDIA IN CHILDREN OF THE FIRST 7 YEARS OF LIFE IN MOLDOVA}

doi:10.1136/archdischild-2012-302724.0827

'S Diacova, II Ababii, 'M Maniuc, 'L Danilov, ${ }^{2} \mathrm{~V}$ Desvignes, ${ }^{3} \mathrm{~N}$ Revenco, ${ }^{10}$ Diacova. ${ }^{1}$ Otorhinolaryngology, Pediatric Clinic, SMPhU Chisinau, Chisinau, Moldova; ${ }^{2}$ Society "Médecins du Monde", Clermont-Ferrand, France; 'PPediatrics Department, SMPhU Chisinau, Chisinau, Moldova

Background Otitis media (OM) is a common problem for primary care, pediatricians and otorhinolaryngologists.

The Aim of our work is to analyze the evolution of OM in children of the first 7 years of life after respiratory tract (RT) pathology.

Subjects Children from 1 to 7 years of life (2785) without evident $\mathrm{OM}$ after standard treatment of RT pathology - bronchitis, pneumonia; RT infection-prone children; chronic nasal breathing difficulties. Healthy children of the same age were included in the control group.

Outcome measures Screening tympanometry, otoscopy; audiology assessment and otomicroscopy for children who failed the screening tests, paranasal sinuses evaluation, otological follow up for children with middle ear pathology during 2 years.

Results We diagnosed OM in $68 \%$ of cases from RT pathology group and in $6 \%$ of cases from control group ( $p$ less than 0.001 ). Acute middle ear effusion was presented in $53 \%$, chronic OM with effusion in $38 \%$, recurrent $\mathrm{OM}$ in $9 \%$. Chronic and recurrent $\mathrm{OM}$ was related with RT infection-prone children ( $p$ less than 0.01 ), age younger than 5 years of life ( $p$ less than 0.01 ) and sinusitis ( $p$ less than 0.01 ). Adhesive and chronic OM was recorded during otomicroscopy and surgery in $12 \%$ of cases.

Conclusions Screening for $\mathrm{OM}$ is important for children with recurrent and chronic RT pathology. These groups of patients need comprehensive diagnostic management and intensive treatment, including surgical one. In healthy children $O M$ is a relative rare and temporary condition.

\section{VITAMIN D STATUS IS NOT ASSOCIATED WITH THE RECURRENT WHEEZY IN INFANCY}

doi:10.1136/archdischild-2012-302724.0828

Y Dallar Bilge, MA Taşar, A Doğan, Fi Arıkan. Pediatrics, Ministry of Health, Ankara Training and Education Hospital, Ankara, Turkey

Low vitamin $\mathrm{D}$ status have been found to be associated with the risk of acute upper and lower respiratory tract infections. The deficiency of vitamin $\mathrm{D}$ was the significant risk factors contributed to the post-bronchiolitis wheezing in children.

We aimed to investigate the association between vitamin $\mathrm{D}$ status and occurrence of recurrent acute bronchiolitiThe children with acute bronchiolitis who aged 2 months to 2 years were hospitalized between December 2008 and April 2009 in the Ankara Training and Research Hospital, Ankara, Turkey were included in the analysis.

This study was prospective and case-control study. We used a questionnaire addressing demographic factors, exposure to sunlight and vitamin use.

We defined children with recurrent wheeze as $=3$ wheezing attacks.

In total, 56 cases and 30 controls were enrolled. The mean age of all children was $12 \pm 7.2$ months ( 2 months- 2 years). The mean of serum $25-\mathrm{OHD} 3$ was $138 \pm 56.7 \mathrm{nmol} / \mathrm{L}$ in total patients, $134.3 \pm 55.6$ $\mathrm{nmol} / \mathrm{L}$ in cases and $145 \pm 59.2 \mathrm{nmol} / \mathrm{L}$ in control group. There was not any significant difference between cases and controls in 25-OHD3 levels ( $p>0.05)$.

The mean of serum $25-\mathrm{OHD} 3$ was $132.4 \pm 58(10-263) \mathrm{nmol} / \mathrm{L}$ in group $1(\mathrm{n}=42)$ and $140 \pm 49(75-208) \mathrm{nmol} / \mathrm{L}$ in group $2(\mathrm{n}=14)$, the difference was not statistically significant $(p>0.05)$. 